\title{
BLOOD TRANSFUSION BY THE CONTINUOUS DRIP METHOD.
}

\author{
By R. A. KING, F.R.C.S.
}

(Assistant to Surgical Unit, St. Mary's Hospital; Assistant Surgeon, Croydon General Hospital.)

A brief resumé of the chief indications for blood transfusion is as follows :-

I. To replace blood lost by hæmorrhage. The more important causes are accidents, hæmatemesis, melæna, obstetrical hæmorrhage, ectopic pregnancy, the pre-operative, operative and post-operative transfusions.

2. To increase the blood volume in surgical shock.

3. To restore blood and to stimulate the hæmopoietic system in anæmias.

4. To restore lost blood in the hæmorrhagic states (hæmophilia and purpura), and to supply certain missing factors, e.g. platelets.

5. To increase the general resistance and immunity in acute and chronic infections by supplying leucocytes and antibodies (immuno-transfusion).

In many of the cases of anæmia, hæmoglobin is so low that transfusion of a pint of blood by ordinary methods is only sufficient to raise the total amount of hæmoglobin by eight or ten per cent. It was originally shown by Marriott and Kekwick that very large quantities of blood can be given by slow constant drip to restore the hæmoglobin to normal levels without increasing the blood volume and over-loading the circulation. Very slow infusion of blood at a rate of about 40 drops a minute raises the hæmoglobin by about ro per cent. in four hours. Excess of plasma is removed from the circulation at about the same rate, the net result being a gain in blood cells and hæmoglobin.

By this method of blood transfusion, over six litres, or eleven pints, of blood have been given continuously over a number of days. Such quantities of blood require the services of several donors, who must all be compatible with the patient and of the same group as one another.

Choice of donor. It is best to obtain a series of donors from among the patient's relatives and friends rather than burden unduly the excellent Red Cross donor service. Each of these volunteers should be in good general health and transmission of syphilis and malaria should be borne in mind. It is essential to prove that patient's and donor's bloods are compatible.

\section{Blood Typing or Grouping.}

Since Landsteiner first discovered three of the four common blood groups (IgOI), and Moss published his group classification (I9IO), blood transfusions have become everyday occurrences. These pioneers showed that certain types of blood, when mixed, were incompatible, and agglutination of corpuscles occurred, causing severe general reactions and sometimes death. They discovered four groups of blood, each of which could be safely mixed with a similar group without agglutination. Choice of appropriate blood groups enables a donor to be used safely for blood transfusion. The following table represents the original Moss classification of groups, and is shown with the modern International classification:

$\begin{array}{lcccc}\text { Moss Groups. } & 1 & 2 & 3 & 4 \\ \text { International Groups. } & \mathrm{AB} & \mathrm{A} & \mathrm{B} & \mathrm{O} \\ \% \text { Incidence (British Isles). } & 4 \% & 45 \% & 10 \% & 41 \%\end{array}$


Method of typing blood. In practice, typing sera of groups A and B are kept as permanent stock, and readily indicate the group of any blood. It is important that the sera should be reasonably fresh and stored in the cold.

A clean glass microscope slide is warmed under the hot tap and dried. It is divided into two compartments, $\mathrm{A}$ and $\mathrm{B}$, with a blue skin pencil, as shown (Fig. I) :

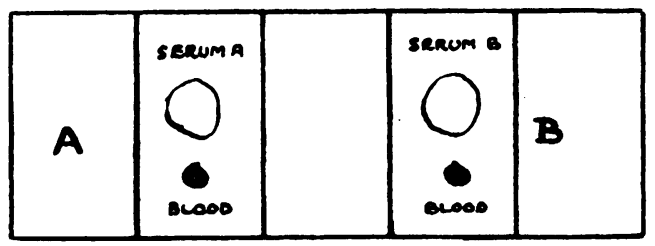

FIG. I.

Two minims of typing serum A are placed in one compartment and the same amount of serum B is placed in the second. Care is taken to label A and B respectively. A drop or two of blood to be tested is placed in each compartment. The slide is now gently tilted to mix blood and serum. Agglutination may or may not occur in either or both compartments. The whole procedure should be carried out in a warm room and the tests observed for about three minutes by holding the slide over a white background. The following are the four possibilities (Fig. 2):
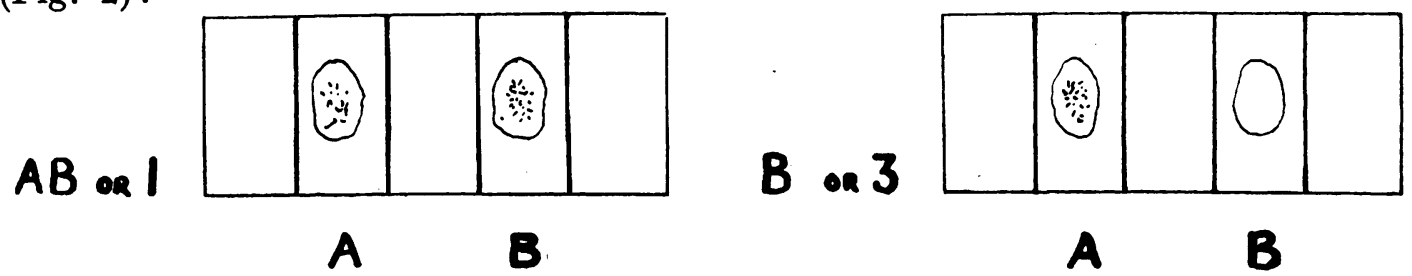

B

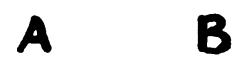

B obtained.

The patient to be transfused is grouped, and a donor of a similar group is

Cross grouping. It is safer to do a further agglutination test on patient's and donor's blood than to transfuse blindly, persons of similar groups. This is an additional check and excludes rare cases of incompatibility.

Macroscopic method.

A c.c. of patient's blood is allowed to clot in a small glass tube. The bead of serum is removed with a capillary tube and blown on to a glass slide, with a similar amount of donor's blood, and observed for at least 7 minutes in a warm 
room. After a time a haze appears, due to clotting. A needle point passed through the blood will lift out the tiny shred of fibrin and allow the test to be observed easily. Agglutination indicates incompatibility, provided the temperature is not too low.

This method is easily carried out, and is quite reliable, but, if circumstances permit, cross grouping done under laboratory conditions gives an absolutely accurate result, as follows:

\section{Laboratory Method.}

It is necessary to have a c.c. or more of the patient's blood and of the donor's blood. Clotting is allowed to occur and the serum is collected in capillary pipettes.

A sample of the patient's red cells is obtained by diluting blood with normal saline, centrifuging and decanting the supernatent fluid. Agglutination is then tested between cells of the donor and the patient's serum, and serum of the donor with the patient's cells. In each test the serum should be diluted with two or three parts of normal saline. The red cells are also added in dilute suspension. The test is incubated in a moist atmosphere at $37^{\circ} \mathrm{C}$. for $\mathrm{I}_{5}$ minutes, and examined under the microscope.

Rouleaux formation in some degree is common, but quite distinct from massive clumping of agglutination. This laboratory method of cross grouping is advisable. in cases of severe toxæmia.

\section{The Collection of Blood.}

Apparatus for the Citrate Method.

1. A container, which should be a wide-mouthed pint bottle, graduated at 100 c.cs, 160 c.cs and 600 c.cs.

2. Sodium citrate solution-3.8 per cent. (isotonic), 160 c.cs in sterile solution.

3. Needle, record fitting, large bore (S.W.G. 13 by $2^{\prime \prime}$ ) and 8 or 9 inches of rubber tubing to fit. N.B.-The needle must be sharp. A needle of slightly smaller bore may be found more convenient by some operators, but the smaller the needle the greater is the likelihood of clotting occurring therein. (Sterilise 1, 2 and 3 in Autoclave.)

4. Record syringe fitted with fine needle and 1 per cent. novocaine.

5. Sphygmomanometer for use as a tourniquet.

\section{Technique of bleeding donor.}

\section{Position.}

It is important to have the donor lying in a good light with the arm extended over a small pillow by his side. Tight neckbands and belts should be loosened, the coat should be removed, and a blanket used as cover. Cooling may cause constriction of the superficial vessels, a process in which the larger veins share. This adds greatly to the difficulty of collecting blood, while warmth has the opposite effect.

Selection of Vein.

In the majority of cases, one of the superficial arm veins of the anti-cubital fossa is used. These are readily visible in most males, but in females may be 
completely hidden in the superficial fat. When distended, these veins, even it invisible, may be identified accurately by careful palpation with the finger tip. In these difficult arms the vein is most readily appreciated at the flexure crease of the elbow, where the skin is thinnest.

In certain cases varicose veins of the lower limb may be used. The leg should hang over the side of a couch on which the donor is lying.

\section{Distention of Veins.}

Preliminary distention of the arm veins a few minutes before collecting blood makes the operation certain and easy. The donor may swing the arm in circles rapidly from the shoulder a few times, then a light tourniquet applied, as high as possible above the elbow. Pressure should be adjusted to cause intense congestion of the arm without reducing the force of the pulse at the wrist. These methods will usually cause sufficient congestion to make the introduction of an intravenous needle a simple matter. Sometimes, however, it may be necessary to warm the donor's limb with an electric cradle or hot water bottle before good veins can be obtained. The tourniquet must be retained in position until the required amount of blood is withdrawn.

\section{Collecting Blood.}

Having distended the veins, the donor's arm and operator's hands are surgically cleaned. A minim only of I per cent. novocaine is injected into the skin at the precise point the intravenous needle is to puncture. Pressure with the left forefinger below the point where puncture is to be made steadies the vein, while the right forefinger and thumb push the needle steadily into the lumen. It is advisable to have washed the needle and rubber tubing previously with some of the citrate solution.

If blood does not run at once the needle should be partially withdrawn and further attempts made to manipulate it into the lumen through the original skin puncture. Should it be necessary to remove the needle, a small gauze pad must be pressed by an assistant over the puncture immediately, otherwise a hæmatoma will rapidly form, because of the raised venous pressure.

Under no circumstances should it be necessary to cut down and expose the vein of a donor for the collection of blood.

Collection is by gravity in the graduated bottle containing I60 c.cs citrate solution, the mouth of which should be covered by a sterile gauze swab. Should the flow slow down, rhythmic flexion and extension of the donor's fingers will restart the flow. Occasionally, slight pressure on the surface of the needle will be necessary to keep the needle aperture away from the side of the vein. In order to ensure thorough mixing of the contents the bottle should be shaken gently at intervals. From 500 to 600 c.cs of blood are taken.

\section{Collection of defibrinated blood.}

\section{Apparatus.}

The container is fitted with a rubber stopper perforated with three holes, through one of which a zig-zag glass rod runs to the bottom, another allows a 
short glass tube to deliver blood into the bottle, the third is used as a vent. (Fig. 3.).

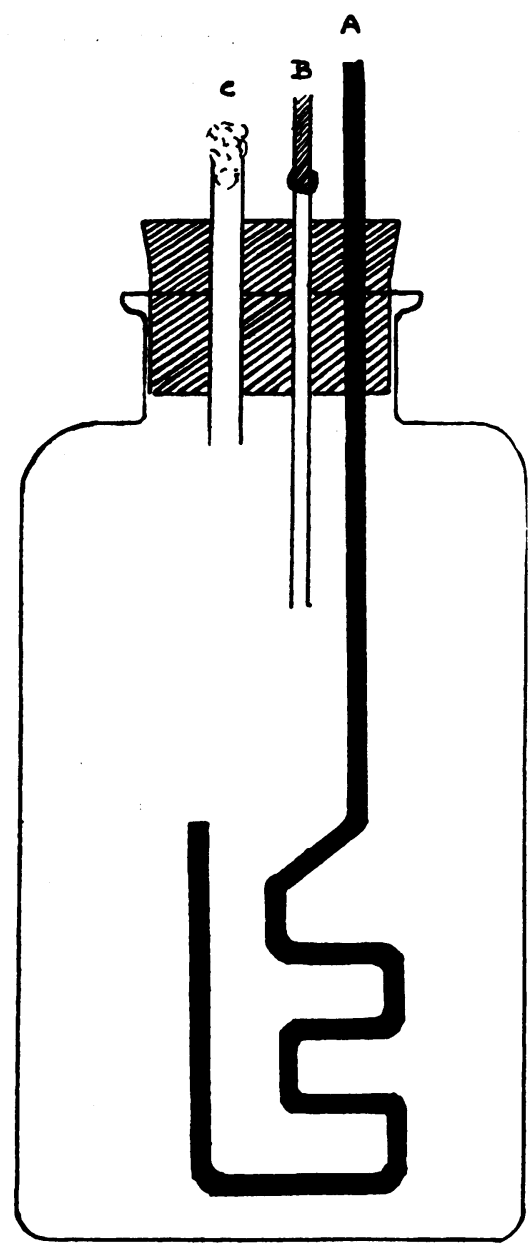

FIG. 3.

DEFIBRINATING BOTTLE.
A. Zig-zag glass rod.
B. Blood tube.
C. Vent with cotton-wool plug.

Technique.

Blood from the donor is allowed to run over the zig-zag. glass rod in the 9 container, which must be kept agitated continuously for about half an hour. This is greatly simplified if the bottle is placed with its base resting on an oiled bench and agitated in a sliding manner in an anti-clockwise direction.

On removal of the glass rod a mass of fibrin is found adherent to it, leaving $\tilde{N}$ fluid blood behind which will not clot. Before use, the blood should be filtered through several layers of fine sterile gauze to remove particles of clot.

Defibrinated blood can be used in the absence of anti-coagulants, and has the advantage that, as these substances are absent, they cannot give any re-action. $\stackrel{\infty}{?}$ 
The donor should remain recumbent for at least half-an-hour after bleeding, and be given plenty of fluids, e.g. hot tea or coffee. A stretched strip of elastoplast, one inch wide, which does not completely encircle the arm, should be applied over a small gauze pad and worn for I2 hours.

\section{Apparatus for Continuous Drip.}

1. Glass reservoir, capacity 1,000 c.cs, fitted with terminal tube and fine metal gauze filter (20 mesh to the inch), and rubber bung with two perforations; through one a short glass tube with a cotton wool filter protrudes; through the other a long glass tube reaches to the base of the reservoir.

2. Rubber pressure tubing, about 6 or $7 \mathrm{ft}$.

3. Lauries Drip Tube (Glass).

4. Needles or Canula (18 or 20 B.W.G. needles).

5. Screw Stopcock.

6. 3 or 4 glass bulb oxygen filters containing cotton wool.

7. Oxygen Cylinder, Reducing Valve, 6 feet rubber tubing incorporating glass filters.

\section{Technique.}

The apparatus is assembled by fixing one end of the rubber tube to the reservoir and other end to needle. It is an advantage to have non-pressure tubing for a few inches at the upper end for the stopcock. A short piece of glass tube inserted in the tubing near the needle is useful as a window. The drip tube should be included towards the upper end below the stopcock. See diagram of apparatus (Fig. 4). An oxygen cylinder with reducing valve and a few feet of rubber tubing (in which are incorporated three wool filters) is connected to a tube leading through the bung to the bottom of the reservoir. An outlet is provided for oxygen by a short glass tube which contains a wool filter and just reaches beyond the bung. All joints should be tied with thread to prevent leaks.

The whole apparatus and oxygen tube should be sterilised in the autoclave or, in an emergency, boiled for half-an-hour in distilled water.

The patient's arm is congested with a sphygmomanometer band, covered with sterile cloths and steadied on a padded back splint. Saline is run into the container until the main tube is filled and about $I^{\prime \prime}$ remains in the bottom; the reservoir should be held high for this. A clip is applied about three inches from the needle.

The apparatus is now ready for the needle to be inserted. As soon as this is done, lower the whole apparatus below the needle level, and undo clip, blood will be seen in the window-tube flowing back towards the saline. As soon as this occurs, raise the reservoir to its working height three to four feet above needle level, and remove the tourniquet.

Three or four strips of adhesive plaster, $3^{\prime \prime} \times I^{\prime \prime}$, fasten the needle and tubing firmly to the forearm. Greater comfort may be obtained if a vein in the middle of the forearm is used, as the splint may be dispensed with, and the patient's movements less hampered.

Blood may now be poured into the reservoir, and the flow adjusted by the stopcock to about 40 drops per minute, or faster if active hæmorrhage is present: 


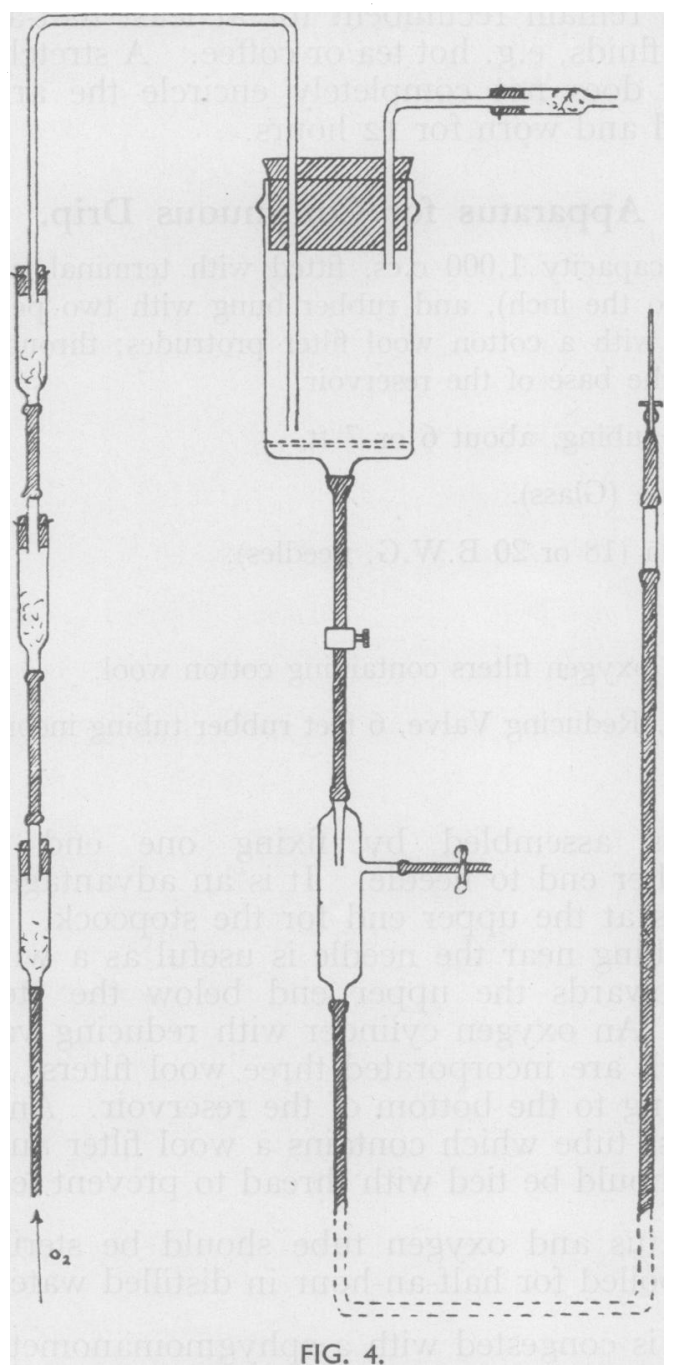

CONTINUOUS DRIP APPARATUS.

(After Marriott \& Kekwick).

The oxygen cylinder is connected to the long tube at the bung, and a slow stream of bubbles passed through the blood.

The rate of this stream should be adjusted between 60-120 per minute, to prevent excessive frothing. The main action of this oxygen stream is to stir the corpuscles and so promote an even flow through the drip tube, at the same time the blood is oxygenated.

It is unnecessary to heat blood when given in this manner. Every effort must $\stackrel{0}{?}$ be made to avoid breathing or speaking over the apparatus, to reduce the risk of $\frac{\varrho}{\Phi}$ droplet infection. 

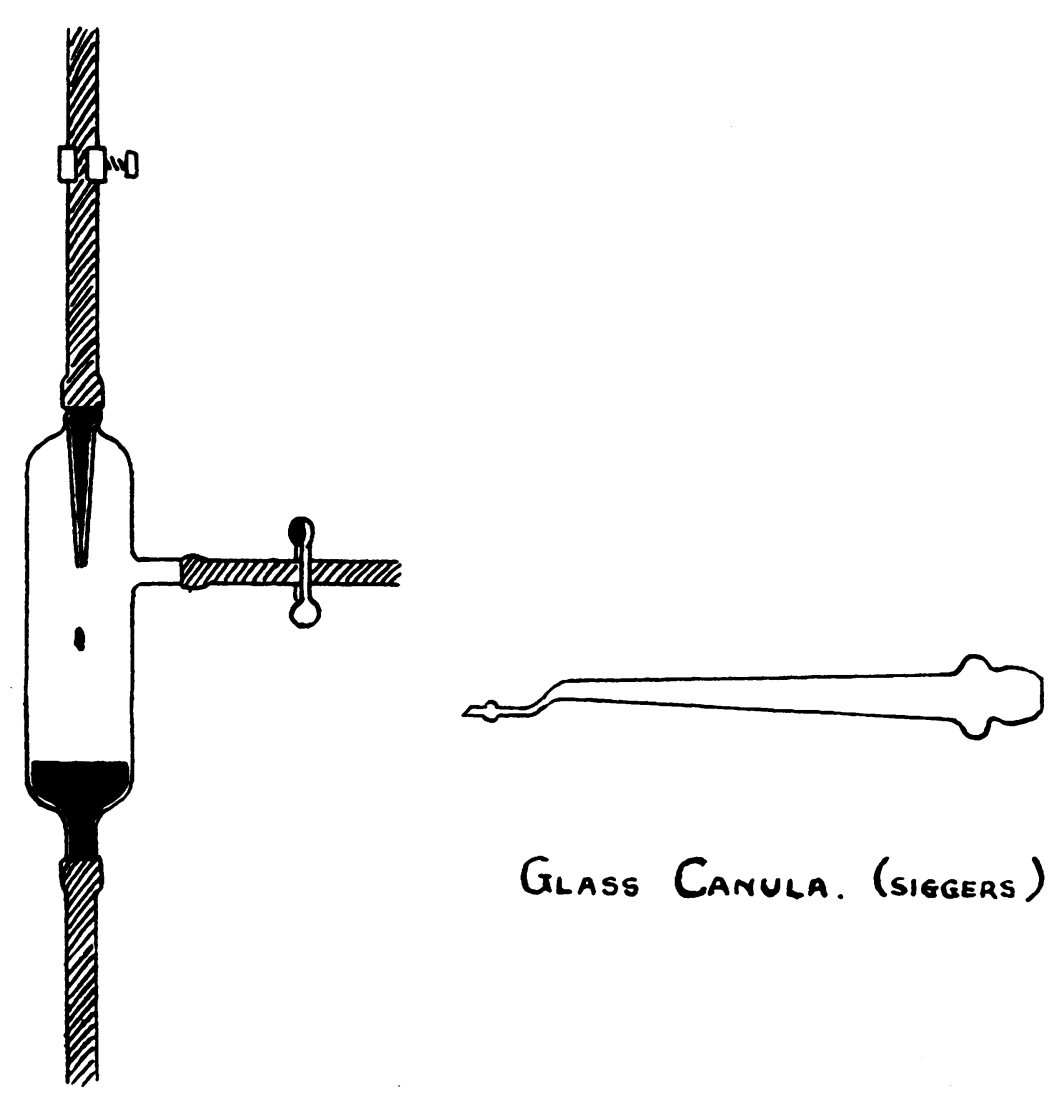

\section{Glass Canula. (siggers)}

\section{Drip Tuge.}

FIG. 5.

\section{Adjustments.}

Height of reservoir from 3-4 feet above arm.

Rate of flow is determined partly by the height of the reservoir, but chiefly by the stopcock.

Drip tube. Blood sometimes wells above the capillary tube and the drip is obscured. A little air should then be allowed to enter by releasing a pinchcock at the side of the drip tube, which allows the level to sink. When the drip tube is not fitted with a pinchcock, the same effect can be obtained by injecting a few c.cs of air, using a record syringe and hypodermic needle to pierce the main rubber tube below the dripper.

Needle. Once the needle is safely in the lumen of a patient's vein, every care should be taken that it does not become dislodged. Sometimes the flow ceases because a minute clot forms in the needle after some hours. This may be dislodged with no harm to the patient by occluding the main rubber tube, an inch or two from the needle, with a pair of artery forceps, and injecting a few c.cs of normal saline into the most distal part of the tube, with a hypodermic syringe. 
Use of canula. In cases of severe collapse one may have to expose a peripheral vein by dissection and tie in a canula. Exposure should be obtained under local anæsthesia, with strict aseptic technique. Through a small transverse incision, not more than half an inch long, the vein is isolated and a double ligature passed beneath with sinus forceps or aneurysm needle. The lower ligature is tied and used to steady the vein by traction, while a small cut is made across the anterior wall of the vein with a pair of fine pointed scissors. One edge of this incision is held by fine dissecting forceps, the canula inserted and tied in by the proximal ligature. A metal or glass canula may be used and connected, as in the case of the needle, to the end of the main delivery tube.

It is an advantage to use one of the branches of the saphenous vein in front of the internal malleolus. This leaves the patient's trunk and arms free to move.

The external jugular vein is quite easy of access also, and can often be used where other peripheral veins are thrombosed or otherwise unsuitable.

The drawback to the use of a canula is the fact that the vein has to be tied and invariably suffers some thrombosis afterwards, whereas it is quite possible to use the same vein half a dozen times if a needle is used instead. However, prolonged transfusion through any vein may cause the onset of thrombosis after twenty-four hours or more. Thus, it may be necessary to change the vein when very large amounts of blood have to be used.

\section{Complications.}

Reaction, in the form of slight rigors, occurs in from $I_{5}$ to 25 per cent. of cases. Careful choice of a suitable donor, use of double distilled water and pure chemicals, does much to lessen these reactions. They are also less common if the blood is given slowly.

Treatment is by liberal application of heat (hot water bottles, or the electric cradle).

Circulatory failure is responsible for the majority of fatalities after transfusion, but this is rare. It may be due to rapid transfusion overloading the circulation and producing odema of the lungs, or it may follow a severe rigor. In all such cases transfusion must cease and an attempt be made to restore the patient by venesection, atropine and cardiac stimulants.

Air Embolism should never occur with proper use of the apparatus. Entry of a c.c. or two of air, however, does no harm.

Suppression of Urine and its attendant dangers may be due to hæmolysis, hæmoglobin forming acid hæmatin in the renal tubules when the urine is acid. This danger is worse where renal disease is present. It can be largely prevented by administration of large doses of alkalis beforehand.

Urticaria and anaphylactic shock have been known to occur where the patient or donor is subject to protein hypersensitivity. 